\title{
The Effects of Extended Input Dynamic Range on Laboratory and Field-Trial Evaluations in Adult Hearing Aid Users
}

DOI: $10.3766 /$ jaaa.18003

\author{
Patrick N. Plyler* \\ Mary Easterday* \\ Thomas Behrens $\dagger$
}

\begin{abstract}
Background: Digital hearing aids using a 16-bit analog-to-digital converter (ADC) provide a 96-dB input dynamic range. The level at which the ADC peak clips and distorts input signals ranges between 95 and $105 \mathrm{~dB}$ SPL. Recent research evaluated the effect of extending the input dynamic range in a commercially available hearing aid. Although the results were promising, several limitations were noted by the authors. Laboratory testing was conducted using recordings from hearing aids set for a flat $50-\mathrm{dB}$ loss; however, field testing was conducted with hearing aids fitted for their hearing loss. In addition, participants rarely encountered input levels of sufficient intensity to adequately test the feature and were unable to directly compare aids with and without extended input dynamic range (EIDR) under identical conditions.
\end{abstract}

Purpose: The effects of EIDR under realistic and repeatable test conditions both within and outside the laboratory setting were evaluated.

Research Design: A repeated measures design was used. The experiment was single-blinded.

Study Sample: Twenty adults (14 males and six females) between the ages of 30 and 71 years (average age 62 years) who were experienced hearing aid users participated.

Data Collection and Analysis: Each participant was fit with Oticon Opn hearing instruments binaurally using the National Acoustics Laboratory-Nonlinear 1 fitting strategy. Participants completed a two-week trial period using hearing aids with EIDR and a two-week trial period without EIDR. The initial EIDR condition trial period was counterbalanced. After each trial, laboratory evaluations were obtained at $85 \mathrm{dBC}$ using the Connected Speech Test, the Hearing in Noise Test, and the acceptable noise level (ANL). Satisfaction ratings were conducted at $85 \mathrm{dBC}$ using speech in quiet and in noise as well as music. Field-trial evaluations were obtained using the abbreviated profile of hearing aid benefit (APHAB). Satisfaction ratings were also conducted in the field at $85 \mathrm{dBC}$ using speech and music. After the study, each participant indicated which trial period they preferred overall. Repeated measures analysis of variances were conducted to assess listener performance. Pairwise comparisons were then completed for significant main effects.

Results: In the laboratory, results did not reveal significant differences between EIDR conditions on any speech perception in noise test or any satisfaction rating measurement. In the field, results did not reveal significant differences between the EIDR conditions on the APHAB or on any of the satisfaction rating measurements. Nine participants (45\%) preferred the EIDR condition. Fifteen participants (75\%) indicated that speech clarity was the most important factor in determining the overall preference. Sixteen participants (80\%) preferred the EIDR condition that resulted in the lower ANL.

*Department of Audiology and Speech Pathology, University of Tennessee Health Science Center, Knoxville, TN; †Centre for Applied Audiology Research, Oticon, Copenhagen, Denmark

Corresponding author: Patrick N. Plyler, Department of Audiology and Speech Pathology, University of Tennessee Health Science Center, 578 South Stadium Hall, Knoxville, TN 37996-0740; Email: pplyler@uthsc.edu

This research was supported by a grant from Oticon.

Poster presentation at the 29th Annual American Academy of Audiology National Convention in Indianapolis, IN, 2017. 
Conclusions: The use of EIDR in hearing aids within and outside the laboratory under realistic and repeatable test conditions did not positively or negatively impact performance or preference. Results disagreed with previous findings obtained in the laboratory that suggested EIDR improved performance; however, results agreed with previous findings obtained in the field. Future research may consider the effect of hearing aid experience, input level, and noise acceptance on potential benefit with EIDR.

Key Words: extended input dynamic range, hearing aid

Abbreviations: $A D C=$ analog-to-digital converter; $A N L=$ acceptable noise level; $A N O V A=$ analysis of variance; $\mathrm{APHAB}=$ abbreviated profile of hearing aid benefit; $\mathrm{BN}=$ background noise; $\mathrm{CST}=$ Connected Speech Test; DSP = digital signal processor; EC = ease of communication; EIDR = extended input dynamic range; GB = global subjective performance; HINT = Hearing in Noise Test; KEMAR = Knowles Electronic Manikin for Acoustic Research; iAGC = input automatic gain control; $\mathrm{LLL}=$ listening at loud levels; NAL-NL1 = National Acoustics Laboratory-nonlinear 1; SNR = signalto-noise ratio; SSQ = speech, spatial, and qualities of hearing scale; UILL = upper input level limit

\section{INTRODUCTION}

$\mathrm{H}$ earing aids have attempted to minimize distortion for many years. Analog hearing aids with linear processing once used peak clipping to limit the maximum output of the device to prevent loudness discomfort for the end user. The disadvantage of limiting hearing aid output with peak clipping was the introduction of harmonic and intermodulated distortion to the output signal, as the distorted output signal could reduce sound quality and speech intelligibility for the listener. As an alternative to peak clipping, compression limiting was used to control the maximum output of the hearing aids. The goal of compression limiting was to minimize distortion in the hearing aid output to improve sound quality and speech intelligibility while protecting the end user from loudness discomfort (Venema, 2017).

Digital hearing aids attempt to minimize distortion as well; however, digital hearing aids process signals differently from analog hearing aids. Both types of hearing aids have similar components, such as microphones and receivers. Digital hearing aids convert the analog input signal from the microphone(s) into a digital format that can be processed by the digital signal processor (DSP) in the hearing aid. This conversion process is accomplished by the analog-to-digital converter $(\mathrm{ADC})$. After processing, digital hearing aids convert the digital output of the DSP into analog format than can be transduced by the receiver into an acoustic hearing aid output. This conversion is accomplished by the digital-to-analog converter.

In order for digital hearing aids to minimize distortion, the acoustic input signal must be accurately reflected in digital format to the DSP during the front-end of the processing stage. If the input acoustic signal is not accurately reflected by the ADC, the processed signal will be distorted, regardless of digital signal processing that is applied (Chasin, 2006; 2012; Baekgaard, Knudsen, et al, 2013; Baekgaard, Rose, et al, 2013). Thus, the ability of the ADC to reproduce the original input acoustic signal can impact the sound quality and clarity of the resulting hearing aid output (Baekgaard, Knudsen, et al, 2013).

Digital hearing aids using a 16 -bit ADC provide a 96dB input dynamic range (Chasin, 2006; 2012; Hockley et al, 2012; Schmidt, 2012; Baekgaard, Knudsen, et al, 2013; Baekgaard, Rose, et al, 2013). In current digital hearing aids, the input level at which the ADC peak clips and distorts input signals ranges between 95 and $105 \mathrm{~dB}$ SPL (Kuk et al, 2014). This suggests that many input signals commonly encountered by hearing aid users should be accurately reflected by the ADC in digital format to the DSP. As a result, hearing aid users exposed to conversational speech ranging in level from $50 \mathrm{~dB}$ SPL (soft) to $80 \mathrm{~dB}$ SPL (loud) are unlikely to perceive any distortion due to ADC peak clipping (Chasin, 2006; 2012; Hockley et al, 2012; Schmidt, 2012; Baekgaard, Knudsen, et al, 2013; Baekgaard, Rose, et al, 2013).

Hearing aid users who frequently experience highlevel input signals, however, may be more susceptible to reduced sound quality and clarity due to ADC peak clipping. For example, hearing aid users may be exposed to music with root mean square intensity levels of 100-105 dB SPL (Chasin, 2006; 2012; Hockley et al, 2012; Schmidt, 2012; Baekgaard, Knudsen, et al, 2013; Baekgaard, Rose, et al, 2013). In addition, intensity levels of shouted speech and music may reach up to 102 and $122 \mathrm{~dB}$ SPL, respectively, if instantaneous peak levels are considered (Oeding and Valente, 2015). Consequently, hearing aid users may experience reduced sound quality and clarity because of ADC peak clipping when attempting to communicate in settings with high input levels such as concerts, sporting events, movie theaters, parties, or crowded restaurants.

One technique currently used to minimize front-end distortion in digital hearing aids is to shift the operating range of the $\mathrm{A} / \mathrm{D}$ converter upward. By shifting the operating range of the $\mathrm{A} / \mathrm{D}$ converter upward, the upper limit of the system above which distortion occurs increases as well (Baekgaard, Knudsen, et al, 2013). Stated differently, the 96-dB dynamic range created 
by the 16-bit ADC may be applied to an input dynamic range of 7-103 dB SPL or it may be shifted upward to an input dynamic range of 17-113 dB SPL.

Oeding and Valente (2015) evaluated the effect of increasing the upper input level limit (UILL) of the ADC on performance in ten experienced hearing aid users both inside and outside of the laboratory setting. For laboratory-based testing, word recognition in noise and sound quality preference were assessed using recordings made on a Knowles Electronic Manikin for Acoustic Research (KEMAR). Recordings were made with a conventional (Widex Clear, 7-103 dB SPL) and a high (Widex Dream, 17-113 dB SPL) UILL hearing aid coupled to the right ear of the KEMAR. The hearing aids were programmed for a patient with a flat $50 \mathrm{~dB}$ HL hearing loss. For word recognition in noise testing, monosyllabic words from the Northwestern University Test Number 6 were presented from $0^{\circ}$ azimuth at three levels: 103, 106, and 109 dBC. Uncorrelated speech-weighted noise was from 90,180 , and $270^{\circ}$ azimuth at $106 \mathrm{dBC}$, thereby resulting in recordings for both hearing aids at three signal-to-noise ratios (SNRs): $-3,0$, and $3 \mathrm{~dB}$. For sound quality preferences, five speech and five music samples were presented in quiet from $0^{\circ}$ azimuth at two presentation levels: $78-83 \mathrm{dBC}$ and $108-113 \mathrm{dBC}$. The participants listened to the recordings via headphones at a level deemed to be tolerable. Results revealed that word recognition in noise was significantly improved for the high UILL condition across each SNR. Sound quality preference results also indicated that participants preferred the hearing aid with the high UILL for speech and noise presented at the higher level.

For testing outside the laboratory, subjective performance was assessed using the Speech, Spatial, and Qualities of Hearing Scale (SSQ) (Gatehouse and Noble, 2004) and the Listening at Loud Levels (LLL) questionnaire (Widex USA-ORCA). Participants were fitted with one set of hearing aids with conventional UILL and one set with high UILL. All hearing aids were programmed using the individual participant's audiogram and the National Acoustics Laboratory-Nonlinear 1 (NAL-NL1) prescriptive approach and were verified with probe microphone measurements. Participants used the hearing aids for a two-month trial period, alternating between hearing aid sets every two weeks. Results revealed no significant differences between the conventional and high UILL hearing aids on the SSQ, the LLL, or overall preference. Thus, Oeding and Valente (2015) concluded increasing the UILL of the ADC improved word recognition and sound quality preferences in the laboratory, but these effects were not evident in more real-world settings.

Although the results from shifting the operating range of the $\mathrm{A} / \mathrm{D}$ converter upward were promising, several limitations were noted by the authors (Oeding and
Valente, 2015). For laboratory testing, word recognition was conducted using recordings from hearing aid sets for a flat $50 \mathrm{~dB}$ loss presented to participants via earphones at listening levels much lower than levels used to obtain the recordings. For field testing, participants were custom fitted with hearing aids appropriate for their hearing loss; however, participants rarely encountered input levels of sufficient intensity to adequately test the feature. Last, listeners were unable to directly compare aids with and without extended input dynamic range (EIDR) under identical listening conditions.

An alternative approach to minimizing front-end distortion in digital hearing aids is to reduce the level of the intensity of the input signal before reaching the $\mathrm{ADC}$, also known as automatic gain control (iAGC). By compressing the input signal, the hearing aid attempts to ensure that the input signal is kept below the distortion limit of the ADC. Recently, a hearing aid was commercially released that uses the iAGC approach to minimizing front-end distortion in digital hearing aids (Dynamic iAGC, Oticon Opn). Although previous research suggested compression limiting may be more beneficial than peak clipping for speech perception for listeners with mild to moderate hearing loss (Dawson et al, 1990; Crain and Van Tasell, 1994) and that compression limiting and peak clipping both degraded sound quality (Stelmachowicz et al, 1999), the authors are unaware of research directly comparing the effects of distortion introduced by either peak clipping or compression at the $\mathrm{A} / \mathrm{D}$ conversion stage. Therefore, the purpose of this research was to determine whether the Dynamic iAGC approach to EIDR improved performance and preference of listeners using hearing aids under realistic and repeatable test conditions both within and outside the laboratory setting. The following research questions were addressed:

- Does the Dynamic iAGC approach to EIDR improve speech perception in noise and listener satisfaction within the laboratory setting?

- Does the Dynamic iAGC approach to EIDR improve subjective performance and listener satisfaction outside the laboratory setting?

- Does the Dynamic iAGC approach to EIDR affect the overall listener preference?

\section{METHODS}

\section{Participants}

Twenty adults participated in this experiment (14 males and six females). The average age of the participants was 62 years with a range of 30-71 years. The experiment was single-blinded. The criteria for inclusion included (a) sensorineural hearing impairment 
consistent with the available fitting range of the test hearing instruments to be used (Figure 1), (b) current hearing aid user, and (c) native English speakers with no known neurological, cognitive, or learning deficits as reported by the participants. Eighteen participants were current users of open-fit behind-the-ear hearing aids, one participant was a current in-the-ear hearing aid user, and one participant was a current completely in the canal hearing aid user. The participants currently used hearing aids from four different manufacturers (1 Bernafon, 2 Starkey, 8 Oticon, and 9 Phonak). A power analysis using a repeated measures analysis of variance (ANOVA) design and assuming large effect sizes $(0.40)$ for each factor showed that 20 participants yielded a statistical power value of 0.99 for EIDR, listening conditions, and abbreviated profile of hearing aid benefit (APHAB) subtests. A large effect size was assumed because small or medium effects may not be relevant clinically. Thus, the sample size in the present study was likely sufficiently large to find a significant effects if a large difference between the variables actually existed. All qualification and laboratory-based testing was conducted in a sound-treated examination room (Industrial Acoustic) with ambient noise levels suitable for testing with ears uncovered (ANSI, 1999). Participants were compensated and the total duration of each experimental session was $<90 \mathrm{~min}$. This study was approved by the Institution Review Board at the University of Tennessee Health Science Center and all participants signed an informed consent form before participation in the study.

\section{Hearing Instruments and Fittings}

Oticon Opn hearing instruments were used in this research. All hearing instruments were open-canal hearing instruments in which an interchangeable receiver unit was placed in the ear canal. The hearing instruments were connected to the receiver using encased wiring and a flexible bend assists with retention of the receiver in the canal. The receiver and dome type were selected using standard clinical procedures, and the same receiver/dome combination was used for both sets of hearing instruments for a given participant. All devices were identical in appearance and features such as noise canceling directional microphones, digital noise reduction, and expansion were activated throughout all experimental testing to maximize ecological validity. Adaptive feedback cancellation was activated in all fittings to maximize high-frequency amplification.

Each participant was fit binaurally with two sets of Oticon Opn hearing instruments. The EIDR feature was activated or $\mathrm{ON}$ for one set of hearing instruments and was deactivated or OFF for the other set of hearing instruments. During the ON condition, Dynamic iAGC was initiated for inputs of $80 \mathrm{~dB}$ SPL or higher to reduce the intensity level of the input signal before reaching the ADC. Dynamic iAGC ensured that the input signal was kept below the distortion limit of the ADC. After the $\mathrm{ADC}$ stage, the reduced gain was added to restore the output to the intended level. As a result, Dynamic iAGC extended the upper limit of the input dynamic range from 95 to $113 \mathrm{~dB}$ SPL for pure tones and from 85 to $103 \mathrm{~dB}$ SPL for speech. (Note: for additional information please consult the Oticon White Paper "The Velox ${ }^{\top M}$ Platform" on the Oticon website). Both sets of hearing aids provided the same processing for inputs $<80 \mathrm{~dB}$ SPL. Audiometric data were used to program each hearing instrument using the NAL-NL1 fitting strategy (Byrne et al, 2001). The hearing instrument parameters were determined by the Oticon software and varied from participant to participant based on their audiometric data. One memory was programmed in each hearing instrument. Each participant used each set of hearing instruments for two, two-week trial periods

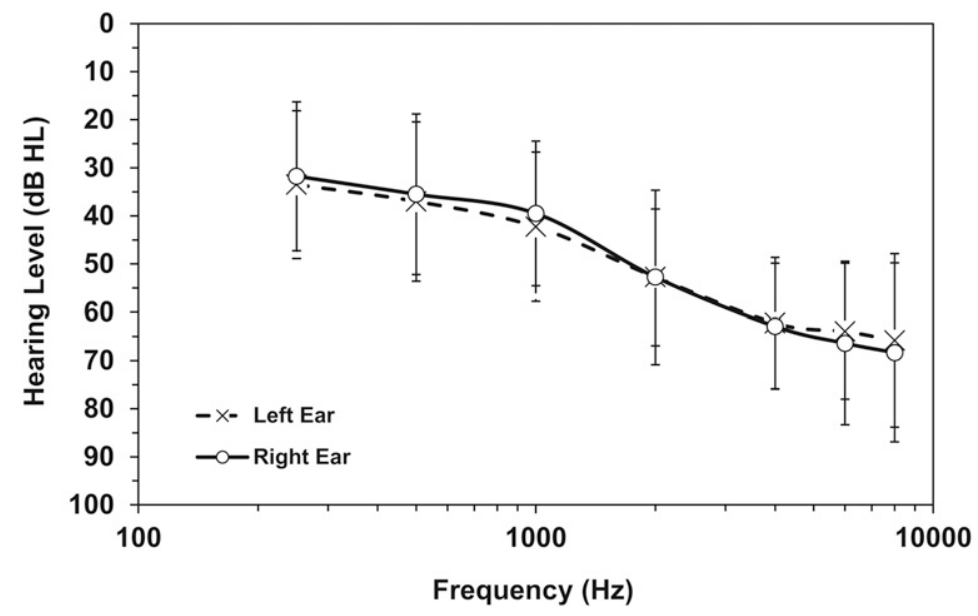

Figure 1. Mean hearing thresholds and standard deviations for left and right ears for participants. 
in a single-blind crossover design. Hearing instruments with EIDR ON were used for one trial period, whereas devices with EIDR OFF were used for the other trial period. The initial amplification condition was counterbalanced between the participants.

Binaural probe microphone measures were conducted before each trial period to verify hearing instrument function. Probe microphone measures were conducted each ear to verify match to NAL-NL1 targets ( $\pm 6 \mathrm{~dB}$ from 500 to $4000 \mathrm{~Hz}$ ) using the Audioscan Verifit Open fittings with Speechmap function at 55, 65, and $75 \mathrm{~dB}$ SPL and at maximum power output. Probe microphone insertion depth was $30 \mathrm{~mm}$ as recommended by Audioscan (Verifit). Data-logging was to be assessed at the end of each trial period; however, problems occurred with the experimental Oticon software used for the study that prohibited obtaining this information.

\section{Laboratory Evaluations}

\section{Speech Perception in Noise}

The Connected Speech Test (CST) (Cox et al, 1987), the Hearing in Noise Test (HINT) (Nilsson et al, 1994), and the Acceptable Noise Level (ANL) test (Nabelek et al, 2004) served as the speech perception in noise stimuli. The CST is a sentence recognition test using everyday connected speech. The CST consisted of 28 pairs of passages ( 24 test and four practice) and each passage pair contains 50 key words. Each passage pair is equivalent in difficulty for listeners with normal hearing (Cox et al, 1987) and hearing impairment (Cox et al, 1988) when presented in background noise (BN) or in quiet (Boike and Souza, 2000). The recommended key word method of scoring was used to determine the percentage of key words correctly identified for each participant. The HINT consisted of 25 lists of 10 English sentences. An adaptive procedure was used to determine the sentence reception threshold in terms of speech-to-noise ratio for each participant using sentence blocks. The standard HINT protocol was used in that noise levels were fixed and speech levels varied based on the participant's response. This procedure ensured that noise levels activated the EIDR feature during testing.

A recording of male running speech (Arizona Travelogue, Cosmos, Inc.) and multi-talker babble (Revised Speech Perception in Noise) served as the stimuli for evaluating acceptance of $\mathrm{BN}$. An adaptive presentation is typically used to determine the most comfortable listening level for speech and the maximum acceptable BN level for each participant. The difference between the most comfortable listening level for speech and the BNL serves as the ANL for each participant (see Freyaldenhoven et al [2005] for review). The ANL protocol used in the present study reflected a slight modification of the original ANL protocol in that noise levels were varied and speech levels remained fixed. This modification ensured that speech levels activated the EIDR feature during testing.

Speech perception evaluations were conducted with the test hearing instruments at the end of the each two-week trial period. All speech stimuli were produced by a compact disc player and routed through a twochannel diagnostic audiometer (GSI-61) to a loudspeaker located at $0^{\circ}$ azimuth in the sound-treated examination room. Background noises were presented through the GSI-61 to a loudspeaker located at $0^{\circ}$ and $180^{\circ}$ azimuth in the sound-treated examination room. The levels of the speech stimuli and BNs were calibrated at the vertex of the listener and checked periodically throughout the experiment.

Participants were seated $1 \mathrm{~m}$ from the loudspeakers located in the sound-treated room. The CST passage pairs were presented at $85 \mathrm{dBC}$ (fixed $5 \mathrm{~dB}$ speech-tonoise ratio). One CST passage pair (50 key words) was administered during each evaluation session and served as the score for that participant in the given session. The HINT was administered using a noise level of $85 \mathrm{dBC}$ and an initial speech level of $80 \mathrm{dBC}$ (adaptive). Two HINT trials were conducted during each evaluation session and the average of the two trials served as the HINT score for that participant in the given session. The ANL was administered using a speech level of $85 \mathrm{dBC}$ and an initial noise level of $65 \mathrm{dBC}$ (adaptive). Two ANL trials were conducted during each evaluation session. An average of the scores for the two trials served as the ANL value for that participant in the given session. Before data collection, an experimental schedule was generated for each participant listing a completely randomized assignment for test order, CST passage pair, and HINT sentence list.

\section{Satisfaction Ratings}

Participants rated their satisfaction regarding sound pleasantness, clarity, and comfort when listening to speech in quiet, speech in noise, and music using a five-point scale ( 1 = very unsatisfied, $2=$ unsatisfied, $3=$ neutral, $4=$ satisfied, and $5=$ very satisfied). Speech in quiet and in noise was assessed using the auditory-visual version of the ANL test (Plyler et al, 2015). The auditory-visual version of the ANL test differs from the traditional ANL test in that it uses an auditory-visual recording of female running speech (Arizona Travelogue, Cosmos, Inc.). Music ratings were conducted using the bluegrass song Rocky Top. Rocky Top is the fight song for the University of Tennessee and was selected because all the participants reported they were familiar with the song and had experienced the song reproduced at high levels. Stimuli levels were consistent with those used for speech perception testing 
to ensure activation of the EIDR feature. Speech in quiet was conducted at $85 \mathrm{dBC}$, speech in noise was conducted at $85 \mathrm{dBC}$ ( $5 \mathrm{~dB}$ SNR), and music was conducted at $85 \mathrm{dBC}$. Satisfaction ratings were obtained twice for each experimental condition at the conclusion of each trial period. An average of the ratings for the two trials served as the satisfaction rating for that participant in the given session. Before data collection, an experimental schedule was generated for each participant listing a completely randomized assignment for test order.

\section{Field-Trial Evaluations}

\section{APHAB}

Subjective evaluations were evaluated by administering the APHAB (Cox and Alexander, 1995) at the end of each two-week trial period. The APHAB is a 24-item questionnaire that assesses subjective hearing instrument benefit in five listening situations or subtests (ease of communication [EC], $\mathrm{BN}$, reverberation, aversiveness to sound, and global subjective performance $[\mathrm{GB}])$.

\section{Satisfaction Ratings}

Participants rated their satisfaction regarding sound pleasantness, clarity, and comfort when listening to speech and music in their home using the same fivepoint scale ( $1=$ very unsatisfied, $2=$ unsatisfied, $3=$ neutral, $4=$ satisfied, and $5=$ very satisfied). Participants were instructed to listen to speech and music at high levels using a television or stereo from their home. Each participant was given a handheld sound-level meter (Extech 407730) to use for the field trial. Participants received hands-on training with the sound-level meter and written instructions for in-home reference. The use of a handheld sound-level meter during the field trial assisted with setting the intensity level to $85 \mathrm{dBC}$ for each rating. Satisfaction rating forms and instructions were also provided to the participants. Satisfaction ratings were conducted five times a week for each two-week trial period; therefore, each participant rated both speech and music a total of 30 times for each trial period (five ratings $\times$ two weeks $\times$ three items). An average of the ratings for the ten trials served as the satisfaction rating for that participant in the given session.

\section{Preference}

At the conclusion of the experiment, each participant was asked to indicate which trial they preferred overall. Participants rank ordered (1-3) the importance the following factors had on determining the overall preference: (a) pleasantness, (b) clarity, and (c) comfort.

\section{RESULTS}

\section{Laboratory Evaluations}

\section{Speech Perception in Noise}

Before statistical analysis, individual percent-correct scores were converted to rationalized arcsine transform units (rau) to stabilize error variance (Studebaker, 1985). Results on the CST, HINT, and ANL were averaged across participants for each EIDR condition (Table 1). Three one-way repeated measures ANOVA were conducted to evaluate the effects of EIDR on each speech perception measurement. The dependent variables were CST score, HINT sentence-recognition threshold, and ANL value. For each ANOVA, the within-subject factor was EIDR with two levels (ON and OFF). The ANOVAs did not reveal a significant main effect for any speech perception measure (Table 2). These results indicated that EIDR did not significantly affect any measure of speech perception in noise.

\section{Satisfaction Ratings}

For satisfaction ratings data, some have argued that individual questionnaire items resulting in ordinal data cannot be accurately evaluated using parametric statistical methods. However, many statistician-scientists disagree with this viewpoint (Velleman and Wilkinson, 1993). Consequently, we have followed the opinion of Nunnally and Bernstein (1994) and used parametric analyses for the satisfaction ratings data. Three twoway repeated-measures ANOVAs were conducted to evaluate the effects of EIDR on listener satisfaction. For each ANOVA, the dependent variables were the satisfaction ratings (pleasantness, clarity, and comfort). The within-subject variables were EIDR with two levels (ON and $\mathrm{OFF}$ ) and listening condition with three levels (speech in quiet, speech in noise, and music). Pairwise comparisons were conducted to further investigate any significant main effects or interactions, whereas familywise error rate was controlled across the tests at the 0.05 level using the Holm's sequential Bonferroni procedure.

Pleasantness, clarity, and comfort satisfaction ratings were averaged across participants for the EIDR and listening conditions (Figures 2-4). For pleasantness and

Table 1. Mean Results for the Speech Perception in Noise Evaluations for Each EIDR Condition

\begin{tabular}{lcl}
\hline Speech Perception Measure & EIDR ON & EIDR OFF \\
\hline CST & $54.0 \%(18)$ & $56.5 \%(23)$ \\
HINT & $3.7 \mathrm{~dB}(2.3)$ & $3.8 \mathrm{~dB}(1.8)$ \\
ANL & $6.4 \mathrm{~dB}(4.3)$ & $7.0 \mathrm{~dB}(6.2)$ \\
\hline
\end{tabular}

Standard deviations are shown in parentheses. 
Table 2. ANOVA Results for the Speech Perception in Noise Evaluations

\begin{tabular}{lccccc}
\hline & $F$ & $\mathrm{df}$ & $p$ & Partial $\eta^{2}$ & $\Omega$ \\
\hline CST & 0.657 & 1.19 & 0.428 & 0.033 & 0.120 \\
HINT & 0.061 & 1.19 & 0.808 & 0.003 & 0.056 \\
ANL & 0.434 & 1.19 & 0.518 & 0.022 & 0.096 \\
\hline
\end{tabular}

clarity, each ANOVA revealed a significant main effect for listening condition; however, the EIDR main effect and the EIDR by listening condition interactions were not significant for either analysis (Table 3). Pairwise comparisons indicated that pleasantness and clarity satisfaction ratings were significantly higher for the speech in quiet and music listening conditions than the speech in noise listening condition. For comfort, the ANOVA did not reveal any significant main effects or interactions. These results indicated that satisfaction with sound pleasantness and clarity was higher when listening to speech in quiet or and music than when listening to speech in noise. These results also indicated that EIDR did not significantly affect any measure of listener satisfaction in any listening condition in the laboratory.

\section{Field-Trial Evaluations}

\section{APHAB}

Scores on each APHAB subtest were averaged across participants for each amplification condition (Figure 5). A two-way repeated measures ANOVA was performed to evaluate the effects of EIDR and APHAB subtest on subjective performance. The dependent variable was the percentage of problems reported on each APHAB subtest. The within-subject factor was EIDR with two levels (ON and OFF) and APHAB subtest with five levels (EC, reverberation, BN, aversiveness to sound, and GB). The analysis revealed a significant main effect for
APHAB subtest (Table 4). Pairwise comparisons were conducted to further investigate the APHAB subtest main effect. Familywise error rate was controlled across the tests at the 0.05 level using the Holm's sequential Bonferroni procedure. Results indicated the percentage of problems for EC was significantly lower than all other subtests. In addition, the percentage of problems for GB was significantly lower than BN. These results indicated that EIDR did not significantly affect the percentage of problems reported for any APHAB subtest.

\section{Satisfaction Ratings}

Pleasantness, clarity, and comfort satisfaction ratings were averaged across participants for the EIDR and listening conditions (Figures 6-8). Three two-way repeated-measures ANOVAs were conducted to evaluate the effects of EIDR on listener satisfaction. For each ANOVA, the dependent variables were the satisfaction ratings (pleasantness, clarity, and comfort). The withinsubject variables were EIDR with two levels (ON and $\mathrm{OFF}$ ) and listening condition with two levels (speech and music). The ANOVA did not reveal any significant main effects or interactions for satisfaction ratings of pleasantness, clarity, or comfort (Table 3). These results indicated that EIDR did not significantly affect any measure of listener satisfaction in either listening condition in the field. Correlational analyses were conducted to determine if satisfaction ratings obtained in the field trial were related to satisfaction ratings obtained in the laboratory (Table 5). Results were significant for

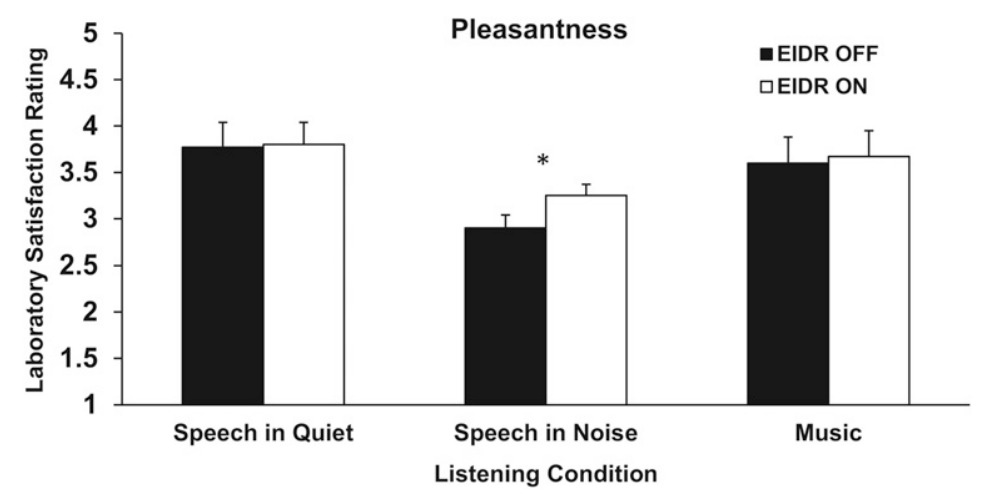

Figure 2. Mean pleasantness satisfaction ratings from the laboratory evaluation for each EIDR and listening condition. Satisfaction ratings were significantly higher for the speech in quiet and music listening conditions than the speech in noise listening condition. Standard deviations are shown. 


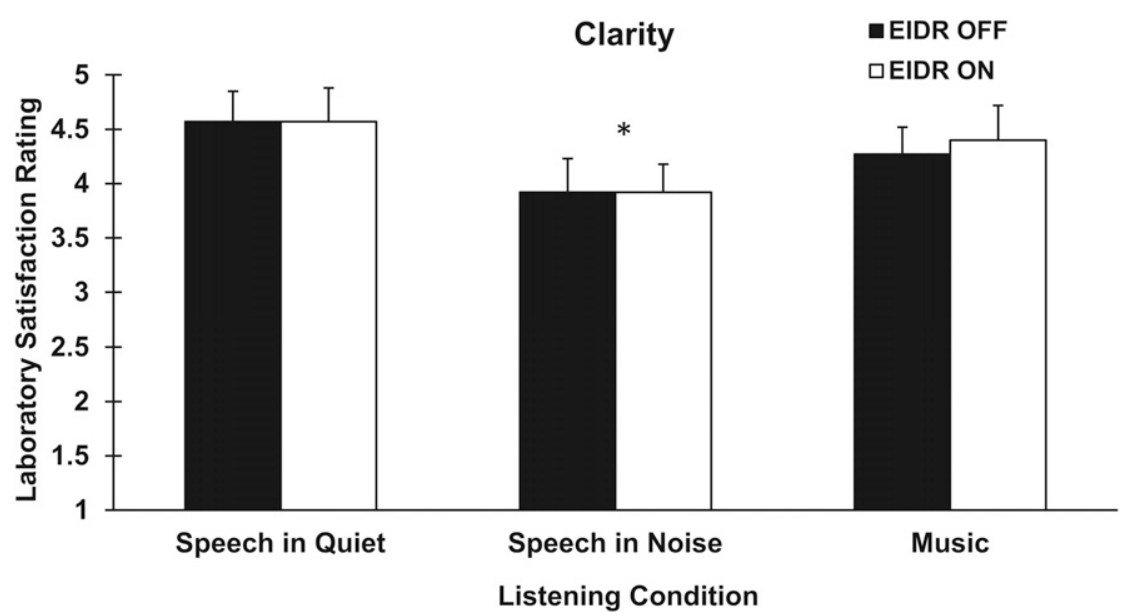

Figure 3. Mean clarity satisfaction ratings from the laboratory evaluation for each EIDR and listening condition. Satisfaction ratings were significantly higher for the speech in quiet and music listening conditions than the speech in noise listening condition. Standard deviations are shown.

both listening (speech and music) and EIDR conditions (ON and OFF) for each satisfaction measure (pleasantness, clarity, and comfort). These results suggested the participants were able to evaluate the hearing aids in the field trial using the personal sound-level meters at levels sufficient to engage the EIDR feature.

\section{Preference}

At the conclusion of the study, each participant indicated which hearing instrument they preferred overall. Preference results were summed across participants and are displayed in Figure 9. A one-sample chi-square test was conducted to determine if hearing aid condition affected the overall preference. The result was not significant $\chi^{2}(1, \mathrm{~N}=20)=0.200, p=0.655$. The proportion of participants that preferred either hearing aid condition was not significantly different than the hypothesized proportion of 0.5 .
Last, participants rank ordered $(1=$ most important and 3 = least important) the importance the following subjective factors had on determining the overall preference: pleasantness, sound clarity, and sound comfort. Importance rankings were averaged within category across the participants for each group (Figure 10). A one-way ANOVA was performed to evaluate the effects of the subjective factors on the overall preference. The dependent variable was the importance ranking for each subjective factor. The within-subject factor was subjective factors with three levels (pleasantness, sound clarity, and sound comfort). The analysis was significant $\left[F_{(2,38)}=14.333, p=<0.000\right.$, partial $\eta^{2}=0.430$, $\Omega=0.998$ ]. Pairwise comparisons indicated that the subjective factor of sound clarity was significantly more important in determining the overall preference than pleasantness or sound comfort. No other comparisons were significant. In addition, sound clarity received a top ranking for 15 of the 20 participants (75\%).

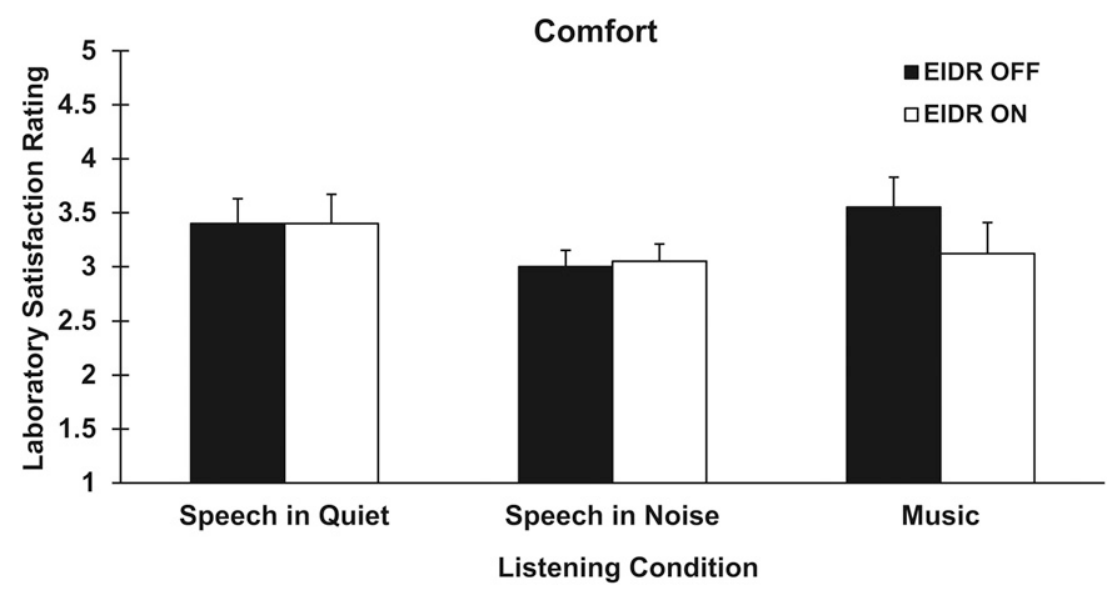

Figure 4. Mean comfort satisfaction ratings from the laboratory evaluation for each EIDR and listening condition. Standard deviations are shown. 
Table 3. ANOVA Results for the Satisfaction Ratings Obtained in the Laboratory and Field-Trial Evaluations

\begin{tabular}{|c|c|c|c|c|c|}
\hline & $F$ & $\mathrm{df}$ & $p$ & Partial $\eta^{2}$ & $\Omega$ \\
\hline \multicolumn{6}{|l|}{ Laboratory } \\
\hline \multicolumn{6}{|l|}{ Pleasantness } \\
\hline EIDR & 1.409 & 1.19 & 0.250 & 0.069 & 0.204 \\
\hline Listening condition & 9.652 & 2.38 & $<0.000$ & 0.337 & 0.973 \\
\hline EIDR $\times$ listening condition & 0.889 & 2.38 & 0.420 & 0.045 & 0.192 \\
\hline \multicolumn{6}{|l|}{ Clarity } \\
\hline EIDR & 0.209 & 1.19 & 0.653 & 0.011 & 0.072 \\
\hline Listening condition & 10.892 & 2.38 & $<0.000$ & 0.364 & 0.986 \\
\hline EIDR $\times$ listening condition & 0.265 & 2.38 & 0.769 & 0.014 & 0.089 \\
\hline \multicolumn{6}{|l|}{ Comfort } \\
\hline EIDR & 0.873 & 1.19 & 0.362 & 0.044 & 0.144 \\
\hline Listening condition & 3.047 & 2.38 & 0.059 & 0.138 & 0.555 \\
\hline EIDR $\times$ listening condition & 2.704 & 2.38 & 0.08 & 0.125 & 0.503 \\
\hline \multicolumn{6}{|l|}{ Field trial } \\
\hline \multicolumn{6}{|l|}{ Pleasantness } \\
\hline EIDR & 0.733 & 1.19 & 0.403 & 0.037 & 0.129 \\
\hline Listening Condition & 2.233 & 1.19 & 0.152 & 0.105 & 0.295 \\
\hline EIDR $\times$ listening condition & 0.905 & 1.19 & 0.353 & 0.045 & 0.148 \\
\hline \multicolumn{6}{|l|}{ Clarity } \\
\hline EIDR & 0.349 & 1.19 & 0.562 & 0.018 & 0.087 \\
\hline Listening condition & 3.610 & 1.19 & 0.073 & 0.160 & 0.438 \\
\hline EIDR $\times$ listening condition & 4.032 & 1.19 & 0.059 & 0.175 & 0.479 \\
\hline \multicolumn{6}{|l|}{ Comfort } \\
\hline EIDR & 3.054 & 1.19 & 0.097 & 0.138 & 0.382 \\
\hline Listening condition & 1.398 & 1.19 & 0.252 & 0.069 & 0.202 \\
\hline EIDR $\times$ listening condition & 0.037 & 1.19 & 0.849 & 0.002 & 0.054 \\
\hline
\end{tabular}

\section{DISCUSSION}

\section{Laboratory Evaluation}

The first purpose of this study was to determine whether the Dynamic iAGC approach to EIDR improved speech perception in noise and listener satisfaction within the laboratory setting. Speech perception in noise outcomes on the CST, HINT, and ANL were not significantly different between the EIDR ON and EIDR OFF conditions. Although listener satisfaction was significantly improved when listening to speech in quiet or to music, listener satisfaction outcomes were not significantly different between the EIDR ON and EIDR OFF conditions for any listening condition. These results indicated that the Dynamic iAGC approach to EIDR did not significantly improve or degrade speech perception in noise or listener satisfaction within the laboratory setting.

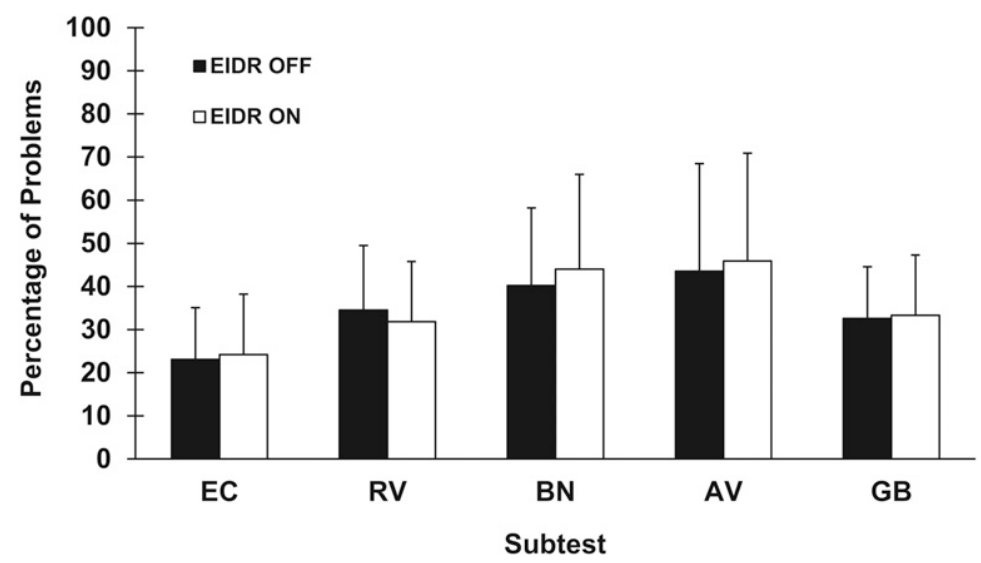

Figure 5. Mean APHAB results for each EIDR condition. The percentage of problems for EC were significantly lower than all other subtests and the percentage of problems for GB were significantly lower than BN. Standard deviations are shown. 
Table 4. ANOVA Results for the APHAB

\begin{tabular}{lccccc}
\hline & $F$ & $d f$ & $p$ & Partial $\eta^{2}$ & \multicolumn{1}{c}{} \\
\hline EIDR & 0.508 & 1.19 & 0.485 & 0.026 & 0.104 \\
Subtest & 9.891 & 4.76 & $<\mathbf{0 . 0 0 0}$ & 0.342 & 0.978 \\
EIDR $\times$ subtest & 0.984 & 4.76 & 0.421 & 0.049 & 0.297 \\
\hline
\end{tabular}

Laboratory evaluation results in the present study were not consistent with previous research. Oeding and Valente (2015) suggested shifting the operating range of the $\mathrm{A} / \mathrm{D}$ converter upward significantly improved word recognition in noise and sound quality judgments within the laboratory setting; however, several limitations were noted by the authors. Methodological differences between the past and present research may explain discrepancies in the results obtained between the two approaches to EIDR within the laboratory. For example, previous research conducted aided word recognition testing and sound quality judgments using recordings made on a KEMAR with hearing aids programmed for a flat 50-dB loss. Recorded stimuli were presented to participants via earphones with a broader bandwidth at listening levels much lower than levels used to obtain the recordings. In the present study, all hearing aids were custom fit to each patient and all stimuli were presented in the sound field via loudspeakers.

Another significant difference between the past and the present research was the input level used. The present study used $85 \mathrm{dBC}$ as the input level, whereas previous research made recordings using inputs of 103 , 106, and $109 \mathrm{dBC}$. For the previous research, inputs may have reached the maximum output of the hearing aids (after UILL) so very little gain would be applied. Thus, it is possible the recorded stimuli did not contain amplification in many frequency regions and only reflected the extended UILL due to the extremely highlevel inputs. For the present study, the inputs were high enough to activate EIDR; however, the hearing aids provided additional amplification in many frequency regions. Consequently, it is possible that the benefit of the EIDR increases if the hearing aid is saturated versus providing additional processing. If true, participants with poorer hearing sensitivity who received the most amplification would be the most likely to reach saturation and thereby benefit from EIDR.

To explore this hypothesis, the pure tone average of 1000,2000 , and $4000 \mathrm{~Hz}$ was calculated for each participant. Benefit from EIDR was then calculated for each experimental condition in the laboratory and the field trial for each participant (EIDR ON-EIDR OFF). Correlational analyses were conducted to determine whether EIDR benefit was related to pure tone average for any experimental condition. Results were not significant for any laboratory of field-trail evaluation. Comparisons were also made between the participant with the best pure tone average ( $40.8 \mathrm{~dB} \mathrm{HL})$ and the participant with the poorest pure tone average ( $72.5 \mathrm{~dB} \mathrm{HL})$. Inspection of the data did not reveal any interesting trends with one exception. The participant with the best hearing reported a $5.5 \%$ increase in the percentage of problems on the Global APHAB subtest when using EIDR, whereas the participant with the poorest hearing reported a $15 \%$ decrease in the percentage of problems on the Global subtest when using EIDR. Although these results suggested that benefit from EIDR was not related to the degree of hearing loss of the participant, this concept could warrant further study in the future.

The present research used standard audiological practice. The domes and amplification used were based

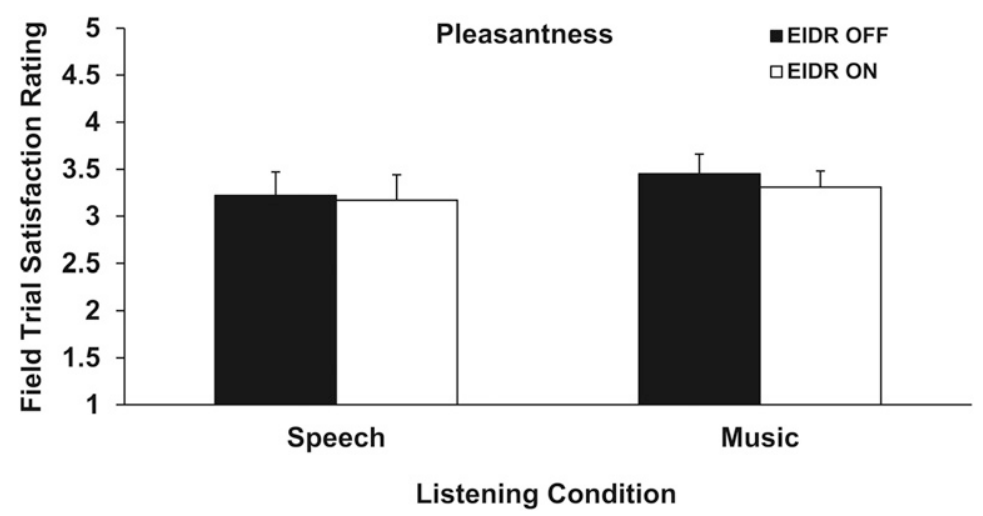

Figure 6. Mean pleasantness satisfaction ratings from the field-trial evaluation for each EIDR and listening condition. Standard deviations are shown. 


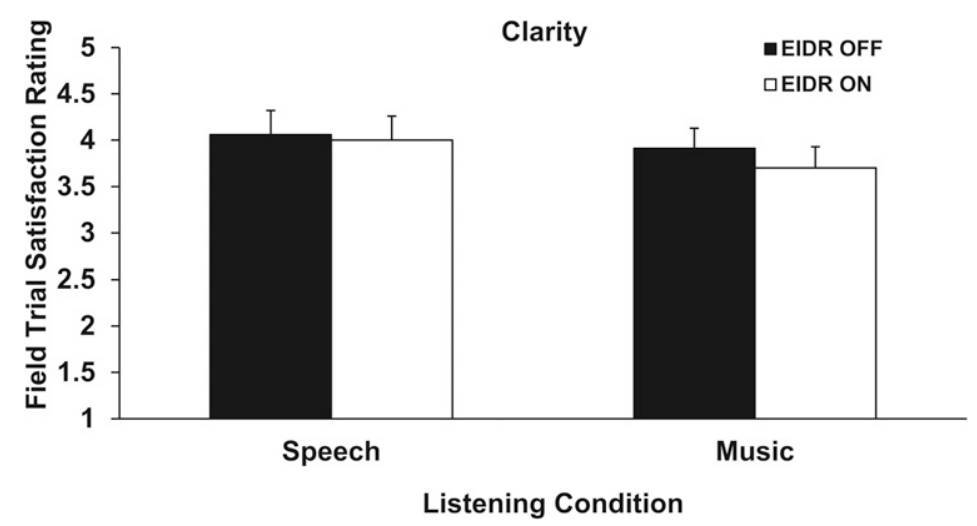

Figure 7. Mean clarity satisfaction ratings from the field-trial evaluation for each EIDR and listening condition. Standard deviations are shown.

on the specific needs of each participant. In previous research, recordings were made using a custom earmold on KEMAR with a 1-mm vent and hearing aids programmed for a $50-\mathrm{dB}$ flat loss. The use of a $50-\mathrm{dB}$ flat loss would allow for low-frequency processing and amplification, whereas more open domes used in standard practice would not. Moreover, previous research used standard procedures with open fittings during their field trial and failed to see benefit with UILL. Thus, it is possible the results of the present study are more reflective of outcomes when standard audiological care is used. Alternatively, EIDR may be more beneficial for patients with more hearing loss in the low frequencies who use more occluding domes. In the present study, seven participants had low-frequency thresholds of $\geq 40 \mathrm{~dB}$ in the low frequencies. Analysis of their data did not support this hypothesis but it may be of interest for future research.

\section{Field-Trial Evaluation}

The second purpose of this study was to determine whether the use of the Dynamic iAGC approach to
EIDR improved subjective performance outside the laboratory setting. Subjective performance outcomes on the APHAB were not significantly different between the EIDR ON and EIDR OFF conditions. Similarly, listener satisfaction outcomes were not significantly different between the EIDR ON and EIDR OFF conditions for either listening condition. These results indicated that the Dynamic iAGC approach to EIDR did not significantly improve or degrade subjective performance or listener satisfaction outside the laboratory setting.

Field-trial evaluation results in the present study were consistent with previous research. Oeding and Valente (2015) reported shifting the operating range of the A/D converter upward did not significantly improve subjective performance on the SSQ or the LLL questionnaire. Methodological similarities between the past and present research may explain congruent outcomes obtained between the two approaches to EIDR outside the laboratory. Oeding and Valente (2015) and the present study both used standard audiological practice when fitting the hearing instruments for the field trial. Thus, differences in the hearing instrument

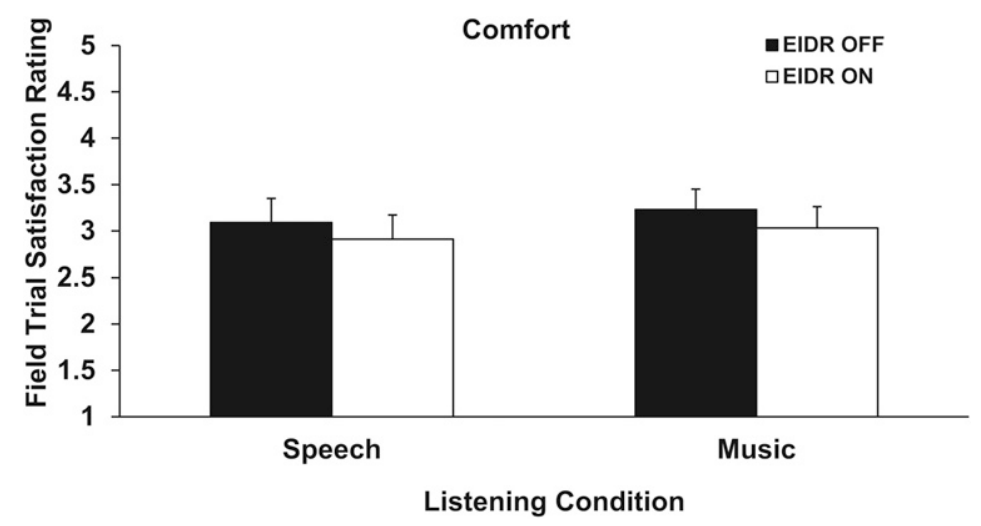

Figure 8. Mean comfort satisfaction ratings from the field-trial evaluation for each EIDR and listening condition. Standard deviations are shown. 
Table 5. Correlation Results between Satisfaction Ratings Obtained in the Laboratory and in the Field-Trial Evaluations

\begin{tabular}{|c|c|c|c|c|}
\hline & \multicolumn{2}{|c|}{ Speech in Quiet } & \multicolumn{2}{|c|}{ Music } \\
\hline & $r(20)$ & $p$ & $r(20)$ & $p$ \\
\hline \multicolumn{5}{|l|}{ EIDR OFF } \\
\hline Pleasantness & 0.703 & 0.001 & 0.783 & $<0.000$ \\
\hline Clarity & 0.803 & $<0.001$ & 0.776 & $<0.0000$ \\
\hline Comfort & 0.870 & $<0.000$ & 0.878 & $<0.000$ \\
\hline \multicolumn{5}{|l|}{ EIDR ON } \\
\hline Pleasantness & 0.772 & $<0.000$ & 0.829 & $<0.000$ \\
\hline Clarity & 0.543 & 0.013 & 0.527 & 0.017 \\
\hline Comfort & 0.726 & $<0.000$ & 0.795 & $<0.000$ \\
\hline
\end{tabular}

fittings that likely contributed to performance measures in the laboratory were not as evident during the fieldtrial evaluations.

In addition, previous research attributed the lack of significant field-trial effects to the possibility the listeners rarely encountered input levels of sufficient intensity to adequately test the feature. In the present research, listeners were trained to use a handheld sound-level meter during the field trial to ensure that satisfaction ratings were conducted at levels of sufficient intensity to engage EIDR. Moreover, satisfaction ratings conducted within the laboratory were significantly correlated with satisfaction ratings conducted in the field trial, thereby suggesting that the participants were able to assess the EIDR feature at sufficient intensity levels. Taken together, the results indicated neither approach to EIDR significantly improved nor degraded subjective performance or listener satisfaction outside the laboratory setting.

\section{Preference}

The third purpose of this study was to determine whether the use of the Dynamic iAGC approach to EIDR affected overall listener preference. Overall listener preference was not significantly different between the EIDR ON and EIDR OFF conditions. In addition, the subjective factor of sound clarity was ranked as the most significant factor in determining the overall preference. Preference results in the present study were consistent with previous research that suggested shifting the operating range of the $\mathrm{A} / \mathrm{D}$ converter upward did not significantly affect the overall preference (Oeding and Valente, 2015).

Although preference results were not statistically significant, participants routinely reported that their preferred strategy was significantly better than the strategy they rejected. Therefore, it was possible that participants that preferred EIDR ON had better results with EIDR ON and vice versa, thereby resulting in similar laboratory and field-trial performance when averaged across participants. In an attempt to further explain overall preference results, participants were regrouped based on the overall preference and their data were reanalyzed to determine whether performance differences existed within each preference group. Audiometric results indicated that listeners in the EIDR ON group had similar hearing loss to listeners in the EIDR OFF group. The pure tone average (500, $1000,2000 \mathrm{~Hz}$ ) was $45.2 \mathrm{~dB}$ HL for the EIDR ON group and $41.6 \mathrm{~dB}$ HL for the EIDR OFF group. Furthermore, the average of $500-8000 \mathrm{~Hz}$ was $51.7 \mathrm{~dB}$ HL for the EIDR ON group and $50.4 \mathrm{~dB}$ HL for the EIDR OFF group.

Examination of the laboratory and field-trial results per preference group revealed trends in the predicted direction for many tests or ratings (Tables 6 and 7). For example, the left side of Tables 6 and 7 indicates listeners that preferred EIDR ON performed better with EIDR ON for many of the tasks; however, the effects remained small and may not be clinically significant. Likewise, the right side of Tables 6 and 7 indicates listeners that preferred EIDR OFF performed better with EIDR OFF for many of the tasks; however, the effects remained small. For the importance rankings,

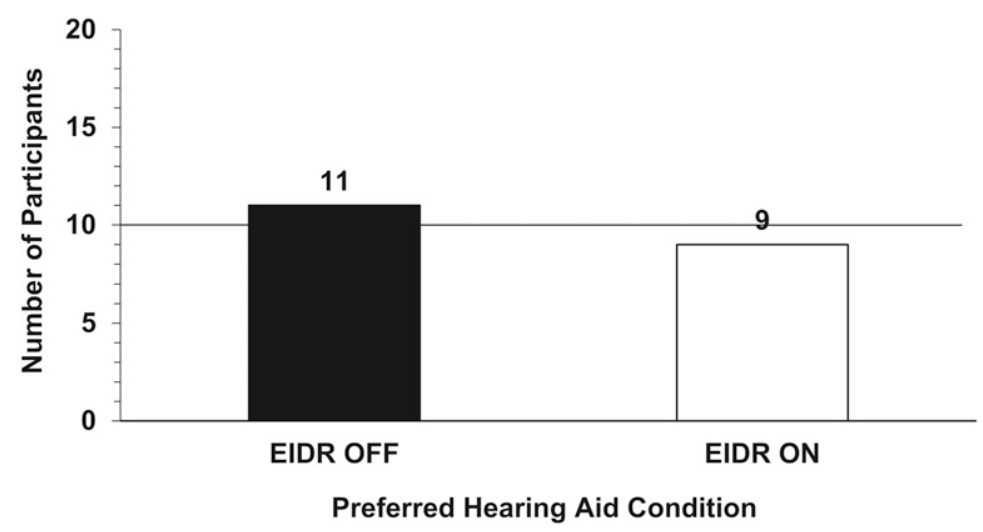

Figure 9. Overall preference results for the EIDR conditions. 


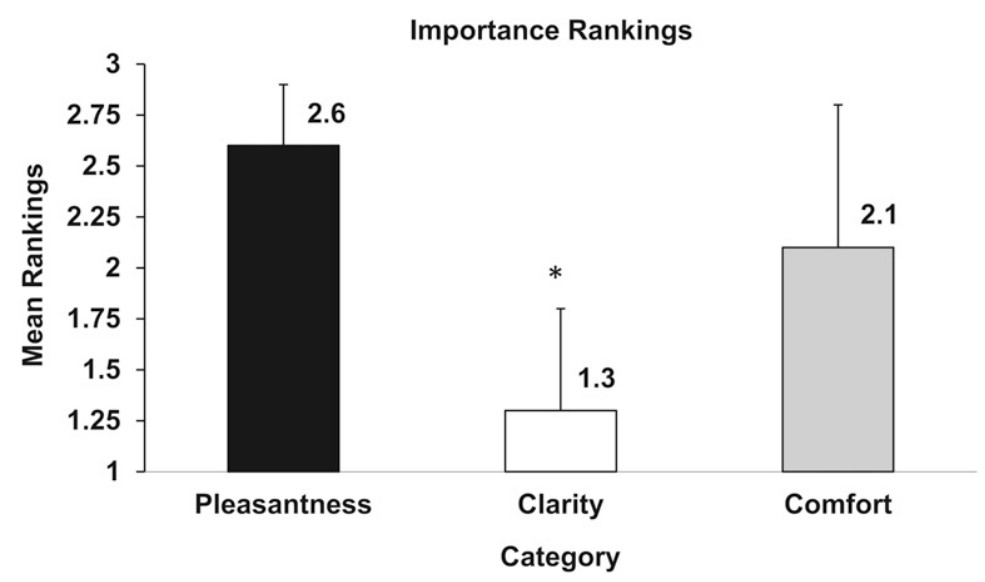

Figure 10. Mean importance rankings regarding overall preference for each subjective factor. Sound clarity was significantly more important in determining overall preference than pleasantness or sound comfort. Standard deviations are shown.

both groups selected sound clarity as the most important factor and sound pleasantness as the least important factor. For the EIDR ON group, importance rankings were clarity (1.2), comfort (2.0), and pleasantness (2.7), whereas the EIDR OFF group importance rankings were clarity (1.3), comfort (2.1), and pleasantness (2.4).

Last, ANL values have been shown to be a good predictor of hearing aid use patterns (Nabelek et al, 2006). Research has demonstrated that individuals who accept BN (low ANL) have a higher probability of becoming successful hearing aid users than individuals who are unable to accept BN (high ANL). The probability of being a successful hearing aid user increases as the unaided ANL value decreases (improves). Furthermore, the ANL model predicted hearing aid use pattern with $85 \%$ accuracy (Nabelek et al, 2006); therefore, ANL values were further examined for each preference group.

Only four of the 20 participants (20\%) preferred the EIDR condition that resulted in the poorer ANL value. Listeners who preferred EIDR ON received an average
ANL reduction of $2.9 \mathrm{~dB}$ when using EIDR ON versus EIDR OFF (Table 6). Conversely, listeners who preferred EIDR OFF received an average ANL reduction of $2.0 \mathrm{~dB}$ when using EIDR OFF versus EIDR ON (Table 6). Interestingly, the average ANL value of the preferred device across the 20 participants was $5.4 \mathrm{~dB}$, whereas the average ANL value of the rejected device across the 20 participants was $7.7 \mathrm{~dB}$. These findings are in agreement with those of Nabelek et al (2006) who reported that the probability of success with hearing aids for a listener with an ANL of $5 \mathrm{~dB}$ is almost $100 \%$, whereas the probability of success with hearing aids for a listener with an ANL value of $7.7 \mathrm{~dB}$ is $\sim 88 \%$. Thus, it is possible the ANL values obtained with each device impacted the overall preference in the present study.

EIDR processing preserves the dynamics of the incoming signal at higher input levels; therefore, participants should encounter peaks at higher output levels with EIDR than without EIDR when listening in high-level

Table 6. Laboratory Evaluation Results for Listeners Who Preferred the EIDR ON or the EIDR OFF Condition

\begin{tabular}{|c|c|c|c|c|c|c|c|}
\hline EIDR ON Group & $\mathrm{ON}$ & OFF & ON-OFF & EIDR OFF Group & ON & OFF & OFF-ON \\
\hline$\overline{\text { CST (\%) }}$ & 50.2 & 52.4 & -2.2 & CST (\%) & 57.1 & 59.8 & 2.7 \\
\hline HINT (dB) & 5.0 & 4.3 & 0.7 & HINT (dB) & 2.8 & 3.5 & 0.7 \\
\hline ANL $(\mathrm{dB})$ & 6.8 & 9.7 & -2.9 & ANL (dB) & 6.0 & 4.0 & -2.0 \\
\hline Pleasantness & & & & Pleasantness & & & \\
\hline Speech in quiet & 3.6 & 3.3 & 0.3 & Speech in quiet & 3.9 & 4.1 & 0.2 \\
\hline Speech in noise & 3.1 & 2.4 & 0.7 & Speech in noise & 3.3 & 3.4 & 0.01 \\
\hline Music & 3.3 & 3.1 & 0.2 & Music & 3.9 & 4 & 0.01 \\
\hline Clarity & & & & Clarity & & & \\
\hline Speech in quiet & 4.4 & 4.4 & 0.0 & Speech in quiet & 4.6 & 4.6 & 0.0 \\
\hline Speech in noise & 3.8 & 3.6 & 0.2 & Speech in noise & 4.0 & 4.1 & 0.1 \\
\hline Music & 4.2 & 4.2 & 0.0 & Music & 4.5 & 4.2 & -0.3 \\
\hline Comfort & & & & Comfort & & & \\
\hline Speech in quiet & 3.0 & 2.8 & 0.2 & Speech in quiet & 3.7 & 3.8 & 0.1 \\
\hline Speech in noise & 2.7 & 2.3 & 0.4 & Speech in noise & 3.2 & 3.5 & 0.3 \\
\hline Music & 2.6 & 2.8 & -0.2 & Music & 3.5 & 4.0 & 0.5 \\
\hline
\end{tabular}


Table 7. Field-Trial Evaluation Results for Listeners Who Preferred the EIDR ON or the EIDR OFF Condition

\begin{tabular}{|c|c|c|c|c|c|c|c|}
\hline$\overline{\text { EIDR ON Group }}$ & $\mathrm{ON}$ & OFF & ON-OFF & EIDR OFF Group & $\mathrm{ON}$ & OFF & OFF-ON \\
\hline$\overline{\mathrm{APHAB}}$ & & & & $\mathrm{APHAB}$ & & & \\
\hline EC $(\%)$ & 23.6 & 25.8 & -2.2 & EC (\%) & 24.6 & 20.9 & -3.7 \\
\hline $\mathrm{RV}(\%)$ & 29.7 & 35.2 & -5.5 & $\mathrm{RV}(\%)$ & 33.4 & 33.9 & 0.5 \\
\hline $\mathrm{BN}(\%)$ & 46.4 & 45.6 & 0.8 & $\mathrm{BN}(\%)$ & 42.0 & 35.7 & -6.3 \\
\hline AV $(\%)$ & 53.4 & 58.8 & -5.4 & AV $(\%)$ & 39.8 & 30.9 & -8.9 \\
\hline GB (\%) & 33.3 & 35.5 & -2.2 & GB (\%) & 33.3 & 30.2 & -3.1 \\
\hline Pleasantness & & & & Pleasantness & & & \\
\hline Speech & 3.1 & 2.9 & 0.2 & Speech & 3.2 & 3.4 & 0.2 \\
\hline Music & 3.0 & 3.1 & -0.1 & Music & 3.5 & 3.7 & 0.2 \\
\hline Clarity & & & & Clarity & & & \\
\hline Speech & 4.2 & 3.9 & 0.3 & Speech & 3.9 & 4.1 & 0.2 \\
\hline Music & 3.7 & 3.6 & 0.1 & Music & 3.6 & 4.1 & 0.5 \\
\hline Comfort & & & & Comfort & & & \\
\hline Speech & 2.8 & 2.8 & 0.0 & Speech & 3.0 & 3.3 & 0.3 \\
\hline Music & 2.8 & 2.8 & 0.0 & Music & 3.2 & 3.5 & 0.3 \\
\hline
\end{tabular}

$\mathrm{AV}=$ aversiveness to sound; $\mathrm{RV}=$ reverberation.

sound environments. Thus, participants who preferred EIDR ON may have done so because of the improved fidelity of the sound, whereas participants preferring EIDR OFF may have made that choice because of increased comfort or pleasantness. In a clinical setting, the improved fidelity and the potentially decreased comfort and pleasantness from the EIDR may be factored in when determining individual preference. It is possible the ANL may have captured that individual preference as $80 \%$ of the participants chose the EIDR condition that provided the better ANL value.

\section{Limitations and Future Studies}

Previous hearing aid experience may have impacted the outcomes of this study. All participants had previous hearing aid experience. It is possible the value of EIDR is more apparent to new users who are not accustomed to listening at such high intensity levels. For experienced hearing aid users who have acclimated to amplification, EIDR may not be that salient; however, the distortion created without EIDR could be more objectionable to users acclimating to hearing aids. Future research should consider the value of EIDR in new hearing aid users.

The benefit from EIDR may also be related to the input level. The present study used input levels of $85 \mathrm{dBC}$ and did not reveal an effect; however, it is possible the real benefit of the feature occurs when input levels are significantly higher and more problematic for the ADC. Moreover, the present study did not use any live music. Live music has a larger input dynamic range than prerecorded stimuli due to compression used in the music industry (Kirchberger and Rosso, 2016). Therefore, future research could determine whether benefit with the feature increases when used in higher input levels or when listening to live versus recorded music. Last, future research should evaluate EIDR in a hearing aid programmed with the feature activated in one memory and deactivated in another memory. This would allow participants to directly assess the relative benefit of the feature daily instead of comparing the absolute performance of the feature across trial periods.

\section{CONCLUSIONS}

7 he purpose of this study was to determine whether 1 the use of the Dynamic iAGC approach to EIDR improved performance and preference of listeners using hearing aids under realistic and repeatable test conditions both within and outside the laboratory setting. The use of EIDR in hearing aids within and outside the laboratory under realistic and repeatable test conditions did not positively or negatively impact objective or subjective performance. Results disagreed with previous findings obtained in the laboratory that suggested EIDR improved performance; however, results agreed with previous findings obtained in the field. Future research may consider the effect of hearing aid experience, input level, and noise acceptance on potential benefit with EIDR.

Acknowledgments. The authors thank Julie Weile, Rowan Lang, and Nicole Humphries for their assistance with the project.

\section{REFERENCES}

American National Standards Institute (ANSI). (1999) Maximum Ambient Noise Levels for Audiometric Test Rooms (ANSI S3.11999). New York, NY: ANSI.

Baekgaard L, Knudsen N, Arshad T, Andersen H. (2013) Designing hearing aid technology to support benefits in demanding situations, part 1. Hear Rev 20(3):42-59.

Baekgaard L, Rose S, Andersen H. (2013) Designing hearing aid technology to support benefits in demanding situations, part 2. Hear Rev 20(6):30-33. 
Boike KT, Souza PE. (2000) Effect of compression ratio on speech recognition and speech-quality ratings with wide dynamic range compression amplification. J Speech Lang Hear Res 43:456-468.

Byrne D, Dillon H, Ching T, Katsch R, Keidser G. (2001) NAL-NL1 procedure for fitting nonlinear hearing aids: characteristics and comparisons with other procedures. J Am Acad Audiol 12(1): $37-51$.

Chasin M. (2006) Can your hearing aid handle loud music? A quick test will tell you. Hear $J$ 59(12):22-24.

Chasin M. (2012) Music and hearing aids-an introduction. Trends Amplif 16(3):136-139.

Cox R, Alexander G, Gilmore C. (1987) Development of the connected speech test (CST). Ear Hear 8:119-126.

Cox R, Alexander G, Gilmore C, Pusakulich K. (1988) Use of the connected speech test (CST) with hearing-impaired listeners. Ear Hear 9:198-207.

Cox RM, Alexander GC. (1995) The abbreviated profile of hearing aid benefit. Ear Hear 16:176-186.

Crain TR, Van Tasell DJ. (1994) Effect of peak clipping on speech recognition threshold. Ear Hear 15(6):443-453.

Dawson P, Dillon H, Battaglia J. (1990) Output limiting compression for the severe-profoundly deaf. Austral J Audiol 13:1-12.

Freyaldenhoven MC, Nabelek AK, Burchfield SB, Thelin JW. (2005) Acceptable noise level as a measure of directional hearing aid benefit. J Am Acad Audiol 16(4):228-236.

Gatehouse S, Noble W. (2004) The speech, spatial and qualities of hearing scale (SSQ). Int J Audiol 43(2):85-99.

Hockley NS, Bahlmann F, Fulton B. (2012) Analog-to-digital conversion to accommodate the dynamics of live music in hearing instruments. Trends Amplif 16(3):146-158.

Kirchberger M, Ross FA. (2016) Dynamic range across multiple genres and the perception of dynamic compression in hearingimpaired listeners. Trends Hear 20:1-16.
Kuk F, Lau C, Korhonen P, Crose B. (2014) Evaluating hearing aid processing at high and very high input levels. Hear Rev 21(3):32-37.

Nabelek AK, Freyaldenhoven MC, Tampas JW, Burchfield SB, Muenchen RA. (2006) Acceptable noise level as a predictor of hearing aid use. J Am Acad Audiol 17(9):635-649.

Nabelek AK, Tampas JW, Burchfield SB. (2004) Comparison of speech perception in background noise with acceptance of background noise in aided and unaided conditions. J Speech Lang Hear Res 47:1001-1011.

Nilsson M, Soli S, Sullivan J. (1994) Development of the hearing in noise test for the measurement of speech reception thresholds in quiet and in noise. J Acoust Soc Am 95:1085-1099.

Nunnally JC, Bernstein IH. (1994) Psychometric Theory. New York, NY: McGraw-Hill.

Oeding K, Valente M. (2015) The effect of a high upper input limiting level on word recognition in noise, sound quality preferences, and subjective ratings of real-world performance. J Am Acad Audiol 26(6):547-562.

Plyler PN, Lang R, Monroe A, Gaudiano P. (2015) The effects of audio-visual stimulation on the acceptance of background noise in listeners with normal and impaired hearing. J Am Acad Audiol 26(5):451-460.

Schmidt M. (2012) Musicians and hearing aid design-is your hearing instrument being overworked? Trends Amplif 16(3): $140-145$.

Stelmachowicz PG, Lewis DE, Hoover B, Keefe DH. (1999) Subjective effects of peak clipping and compression limiting in normal and hearing-impaired children and adults. J Acoust Soc Am 105(1):412-422.

Studebaker GA. (1985) A "rationalized" arcsine transform. J Speech Hear Res 28:455-462.

Velleman PF, Wilkinson L. (1993) Nominal, ordinal, interval, and ratio typologies are misleading. Am Stat 47:65-72.

Venema TH. (2017) Compression for Clinicians: A Compass for Hearing Aid Fittings. San Diego, CA: Plural Publishing. 